\title{
An analysis of working days contamination in micropulsation measurements
}

\author{
Umberto Villante and Massimo Vellante \\ Dipartimento di Fisica, Università dell'Aquila and Area di Astrogeofisica, L'Aquila, Italy
}

\begin{abstract}
A long term analysis of the power of geomagnetic variations in the micropulsations band at L'Aquila (Italy) revealed a weekly variation represented by an increased level of power in the working days with respect to Sundays. The maximum increase, by a factor of $\sim 1.2$, occurs during morning hours in the $100-500 \mathrm{~Hz}$ band. A daily-light-saving time effect has been also identified. Some implications on a wider scientific context are
\end{abstract}

Key words geomagnetic pulsations - man-made noise

\section{Introduction}

In recent years it has become more and more evident that careful analysis of geomagnetic micropulsations in the approximate ULF frequency range $0.001-10 \mathrm{~Hz}$ may be interesting for several aspects of geophysical sciences. In particular, as discussed in several papers (Samson, 1991, and papers referenced therein), micropulsation measurements, which allow interesting investigations of important features of the interaction between the solar wind and the Earth's magnetosphêre, also provide information on the physical characteristics of the magnetospheric and ionospheric plasma populations. On the other hand, many geophysical investigations are based upon the study of the electromagnetic signals, in the sense that a

Mailing address: Dr. Massimo Vellante, Dipartimento di Fisica, Università dell'Aquila, Via Vetoio 10, 67010 Coppito, L'Aquila, Italy; e-mail: massimo.vellante @aquila.infn.it recording of the natural electromagnetic waves that fill our environment represents a useful tool for studying the characteristics of our planet. In this sense, geomagnetic field variations in the frequency range of micropulsations may provide useful information on the local structure of the Earth's crust (Meloni et al., 1985; Pilipenko and Fedorov, 1993; Villante et al., 1998). Growing attention, moreover, has been paid in the last several years to possible relations connecting the local increasing level of the micropulsation energy (within $\sim 100 \mathrm{~km}$ ) with the earthquake occurrence (Fraser-Smith et al., 1990, 1994; Molchanov et al., 1992). Lastly, some correspondence between biological variations and solar and geophysical activity has recently been speculated (Breus et al., 1995), and special attention has been paid to the possible influence of natural and artificial ULF electromagnetic fields on human health (Ptitsyna et al., 1996, and papers referenced therein).

In the present paper we have conducted a long term, statistical analysis of micropulsation measurements performed at L'Aquila (Italy, $L=1.6, \mathrm{LT}=\mathrm{UT}+1)$ since 1985 to determine whether local man-made disturbances may par- 
tially affect experimental observations. This kind of analysis may be interesting in a wide scientific context: indeed, according to previous arguments, for several applications it might be useful to perform ULF measurements not too far from densely populated areas where it is important to make a clear distinction between natural and artificial signals.

As discussed by Villante and Vellante (1997), at our latitudes the daytime micropulsation activity is found to be mostly characterized by the occurrence of two dominant oscillation frequencies, $f_{1}$ and $f_{2}$, which can be interpreted in terms of a concurrent action of an external driving source of waves (related with upstream waves generated in the foreshock region) with a wide spectrum tipically peaked at $f_{1}$, and a local signal amplification at the frequency $f_{2}$ of the fundamental field line oscillation. As shown by Vellante et al. (1996), both these frequencies experience a significant solar cycle variation in the period of interest (1985-1994): in particular, while $f_{1}$ was observed to increase approximately from $35 \mathrm{mHz}$ (solar minimum, 1985,1994 ) to $50 \mathrm{mHz}$ (solar maximum, 19891991), $f_{2}$ decreased approximately from $80 \mathrm{mHz}$ (solar minimum) to $60 \mathrm{mHz}$ (solar maximum). Amplitudes of these quasi-monochromatic signals tipically overcome the noise level discussed in the present investigation at least by a factor of 10 .

\section{Experimental apparatus and data analysis}

The micropulsation measuring system is located in a quiet place approximately $10 \mathrm{~km}$ out of the town of L'Aquila (Central Italy) $\sim 50 \mathrm{~km}$ from the closest electric railway. As discussed by Cantarano et al. (1983), it basically consists of three high sensitivity search coil magnetometers. Sensors are oriented along geographic north-south $(X)$, east-west $(Y)$, and vertical $(Z)$ directions. The amplitude response of the instrument is approximately proportional to the frequency for $f$ smaller than $0.3 \mathrm{~Hz}$. This behaviour accounts for the expected $f^{-1}$ dependence of the field magnitude of natural signals and reduces the dynamic spectrum of the out- put signal. On the high frequency side a rapid decrease of the transfer function removes the aliasing effects of the higher frequency components. A data processing unit samples the signals at a rate of $16 \mathrm{~Hz}$, low-pass filters and stores the data at a sampling frequency of $1 \mathrm{~Hz}$.

The time interval analyzed in the present investigation extends from January 28, 1985 to September 30, 1994 with a large data gap from January 1, 1986 to July 30, 1987. The data coverage after this data gap amounts to about $80 \%$ of the total time. Power spectra of the geomagnetic field components were computed for local daytime hours (0500-1700 UT) over consecutive 4 min intervals. The same kind of analysis conducted for night-time intervals did not show any clear evidence for the effects discussed in the present investigation. The spectra were evaluated in the frequency range 4-500 $\mathrm{mHz}$, with a sampling of $4 \mathrm{mHz}$, by using the maximum entropy method with an autoregression order equal to 30 . We then computed 28060 hourly average spectra for the whole time interval. For the present investigation we further integrated each hourly spectrum over frequency ranges which approximately correspond to the usual Pc1-Pc4 classification scheme. In addition, we separated the nominal Pc3 band into two different frequency intervals in which, as previously reminded, $f_{1}$ and $f_{2}$ events would be preferentially expected. As a matter of fact, the results discussed hereafter are organized in the following frequency bands: $P c 1 \quad(200-500 \mathrm{mHz}), P c 2 \quad(100-200 \mathrm{mHz})$, Pc3a $(45-100 \mathrm{mHz}), P c 3 \mathrm{~b}(20-45 \mathrm{mHz}), P c 4$ (5-20 mHz).

\section{Experimental results}

Man-made disturbances are in general expected to perturb electromagnetic measurements on a wide range of temporal scales (at least from daily to seasonal). On the other hand, natural signals are also expected to show daily, seasonal and solar cycle variations (Jacobs, 1970). We found it useful to focus our attention on the analysis of weekly variations (if any) which obviously might be better related to artificial effects. 

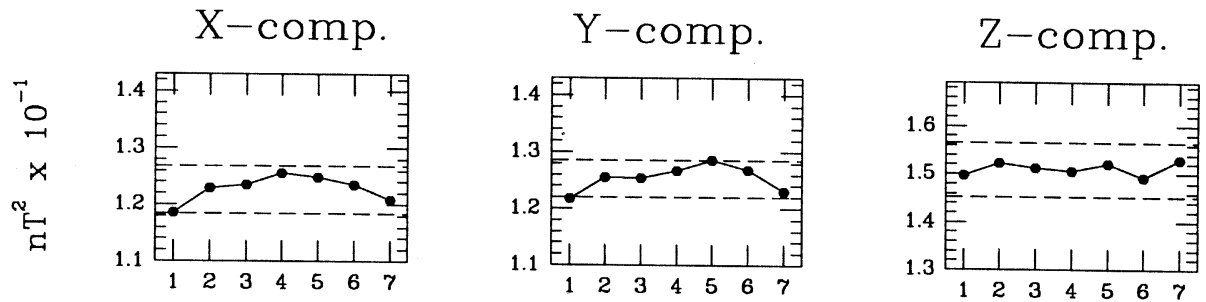

Pc4
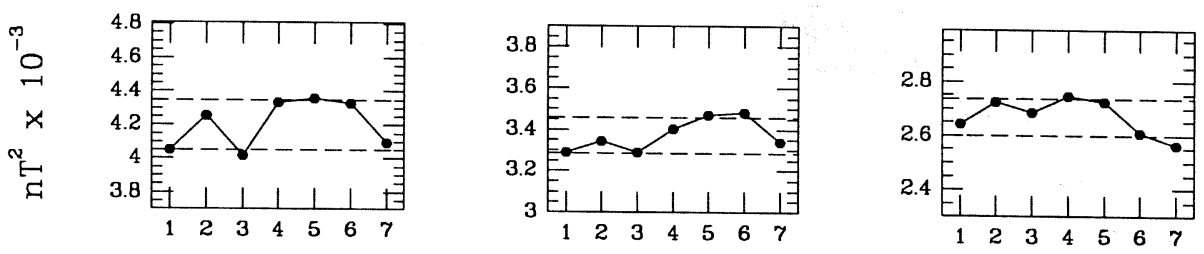

Pc3b
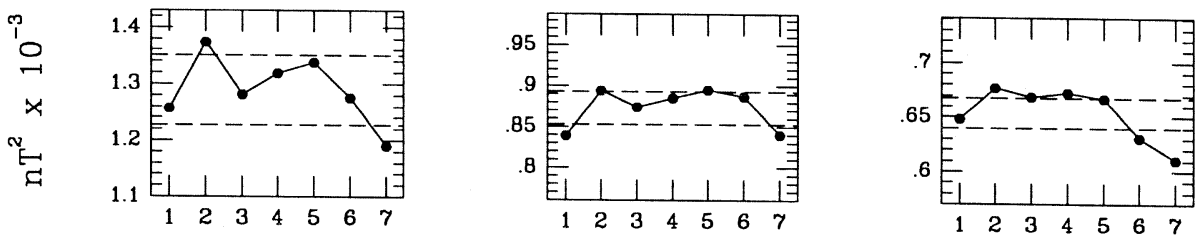

Pc3a
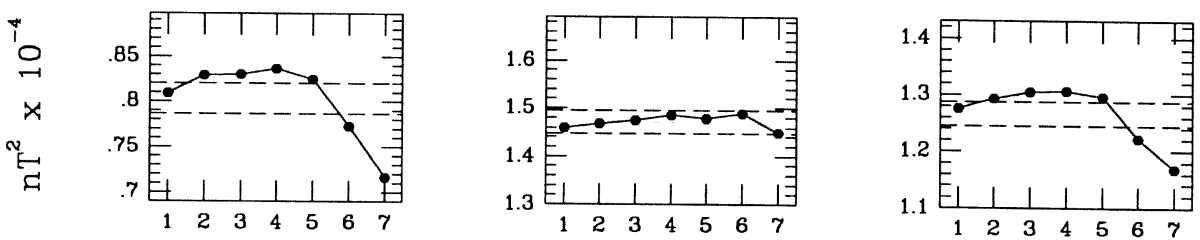

Pc2
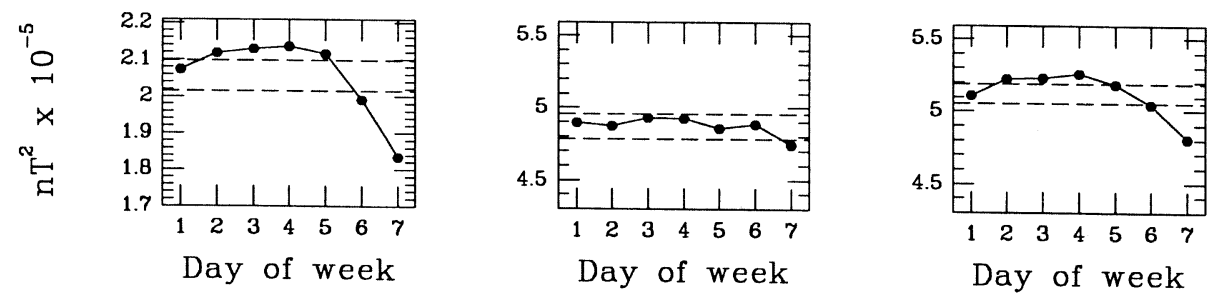

Pc1 Fig. 1. Weekly variation of the average power of magnetic field components in different frequency bands.
Dashed lines identify the $99 \%$ confidence interval.

The average power values for the whole interval as organized in terms of week days (Monday $=1$ ) are shown in fig. 1. These averages were obtained after removing, for each frequency band and for each component, the $5 \%$ highest power values; as a matter of fact, this procedure accounts for extreme power levels associated with peculiar events such as geomagnetic storms and highly localized artificial disturbances. After that, the number of hourly powers concurring to average values in fig. 1 ranged between 3600 and 4000. Dashed lines 

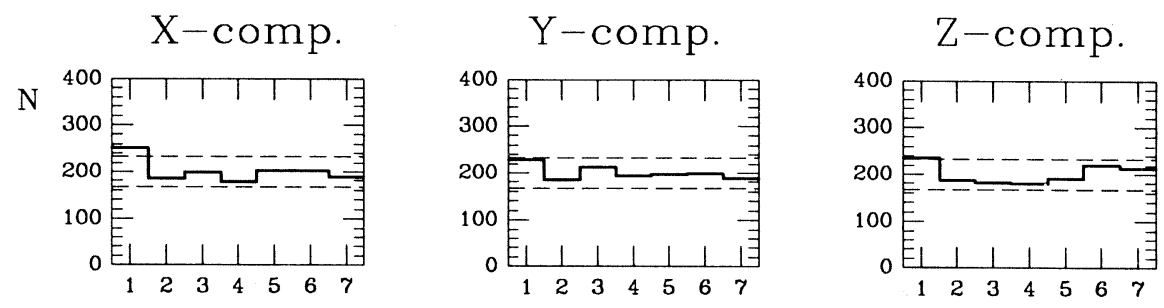

\section{Pc4}
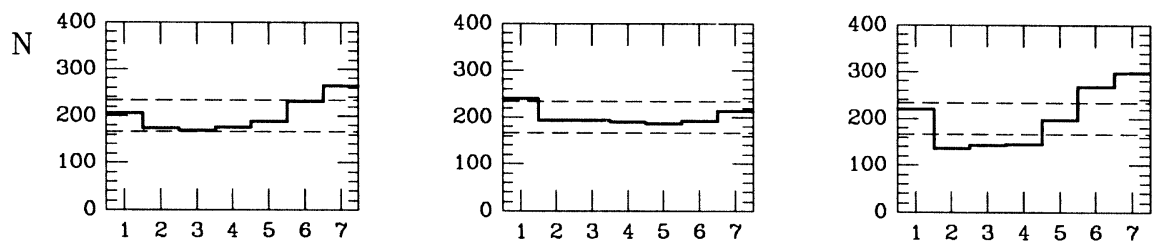

Pc3b
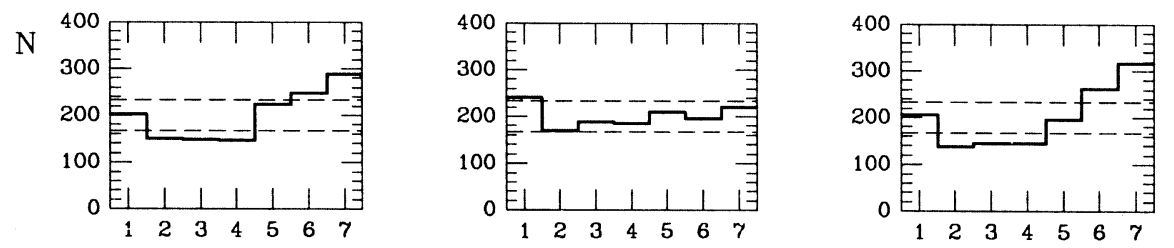

Pc3a

$\mathrm{N}$
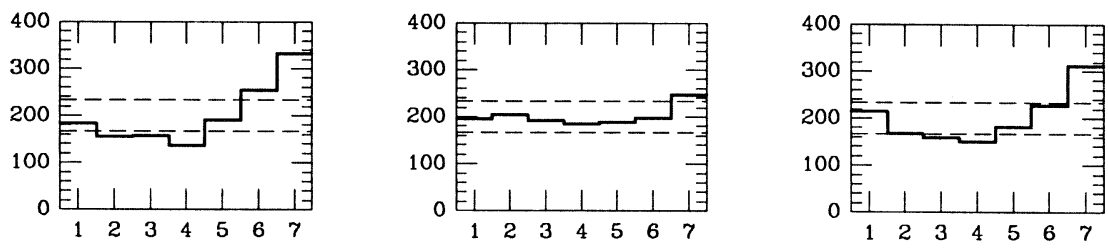

Pc2
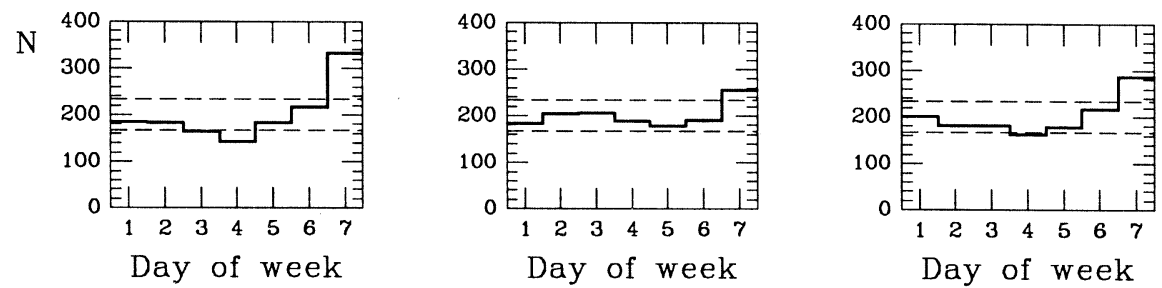

Pc1

Fig. 2. Weekly distribution of the lowest values of power (5\% of the whole population) in different frequency bands. Dashed lines identify the $99 \%$ confidence interval.

define the region of $99 \%$ occurrence for 3800 points averages computed from randomly selected hourly powers.

In addition, in order to provide approximately the same visual representation for equal relative variations of experimental measure- ments, we chose vertical limits in a constant ratio (1.3) for each panel.

The results obtained for the highest frequency bands $(P c 1-P c 3 a)$ reveal a significant weekly modulation of the powers which provides minimum values on Sundays together 
with a significantly reduced power on Saturdays. Some evidence for depressed Monday values can be also identified in most cases. In the lower frequency bands $(P c 3 \mathrm{~b}-P c 4)$ some of the features observed at higher frequencies can still be identified, although relative variations are in general smaller and within confidence limits. Separate analysis conducted for different years confirms that previous effects are a permanent feature at our station with a Sunday minimum which always characterizes the highest frequency powers. All the effects appear also more pronounced in the $X$ and $Z$ components.

Figure 2 shows the results of an independent test which reinforce the conclusions of the previous paragraph. We selected, for each component and frequency band, the $5 \%$ of hourly intervals which correspond to lowest power values. Their distributions versus week days in fig. 2 (dashed lines identify now the theoretical $99 \%$ confidence interval for a flat occurrence probability) show (except for the lowest frequency band) that the lowest power levels are mostly associated to weekends for the $X$ and $Z$ components and also provide clear evidence for the Sunday effect in the highest frequency bands of the $Y$ component.
In order to better identify the occurrence of the man-made disturbances we also conducted the same analysis as in fig. 1 for morning and afternoon observations. The results of fig. 3 which refer to the $P c 2$ band (similar results are obtained for the $P c 1$ band) show that the weekly excursion is more pronounced during the morning hours. In particular, during the morning, the average excess of power from Tuesday through Friday («working day noise») with respect to Sunday is of the order of $20 \%$ and $15 \%$ for the $X$ and $Z$ components respectively. The results in fig. 3 also show that the effect of reduced power on Mondays is a morning feature.

Lastly, a more detailed analysis was conducted for each daytime hour separating winter (October through March) from summer (April through September) months. Figure 4 shows, for the $P c 2$ band, the difference between the working days average power and the Sunday average power versus daytime hour. As it can be seen, the working day noise characterizes all daytime hours. It is also worth noting that peak values occur at 8 UT during winter months and at 7 UT during summer months, i.e. at the same time if we take into account the introduction of the daily-light-saving time during the summer months in Italy.
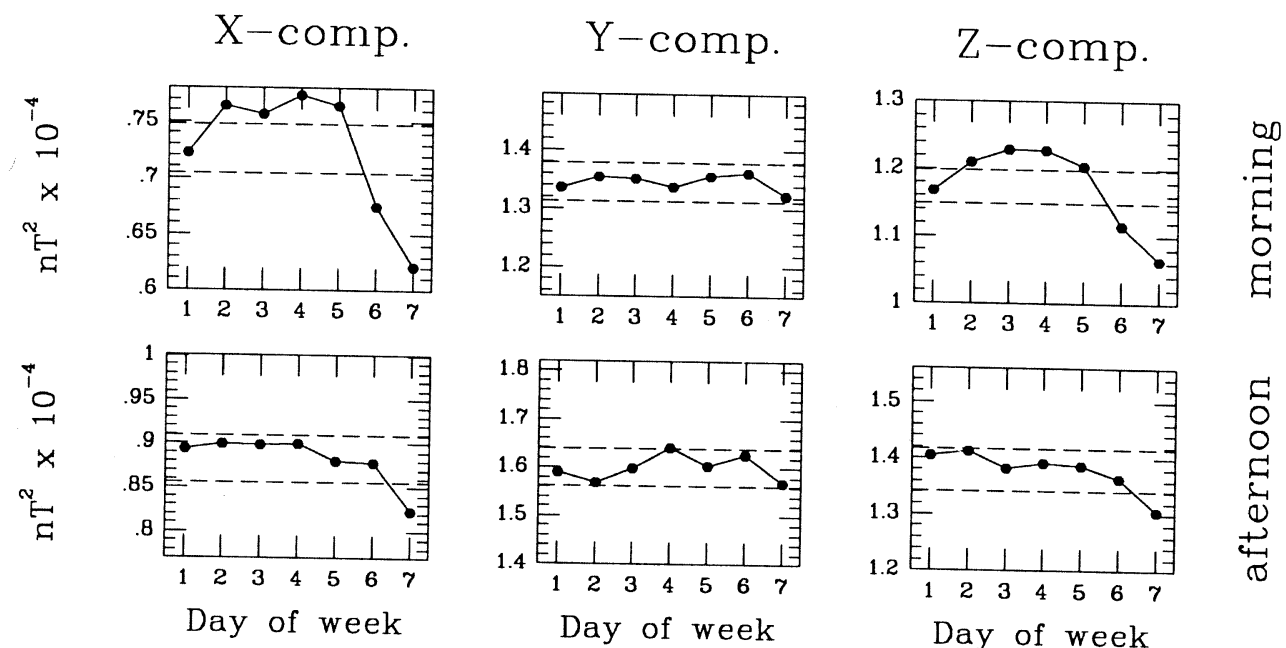

Fig. 3. Weekly variation of the $P c 2$ average power for morning and afternoon observations. 

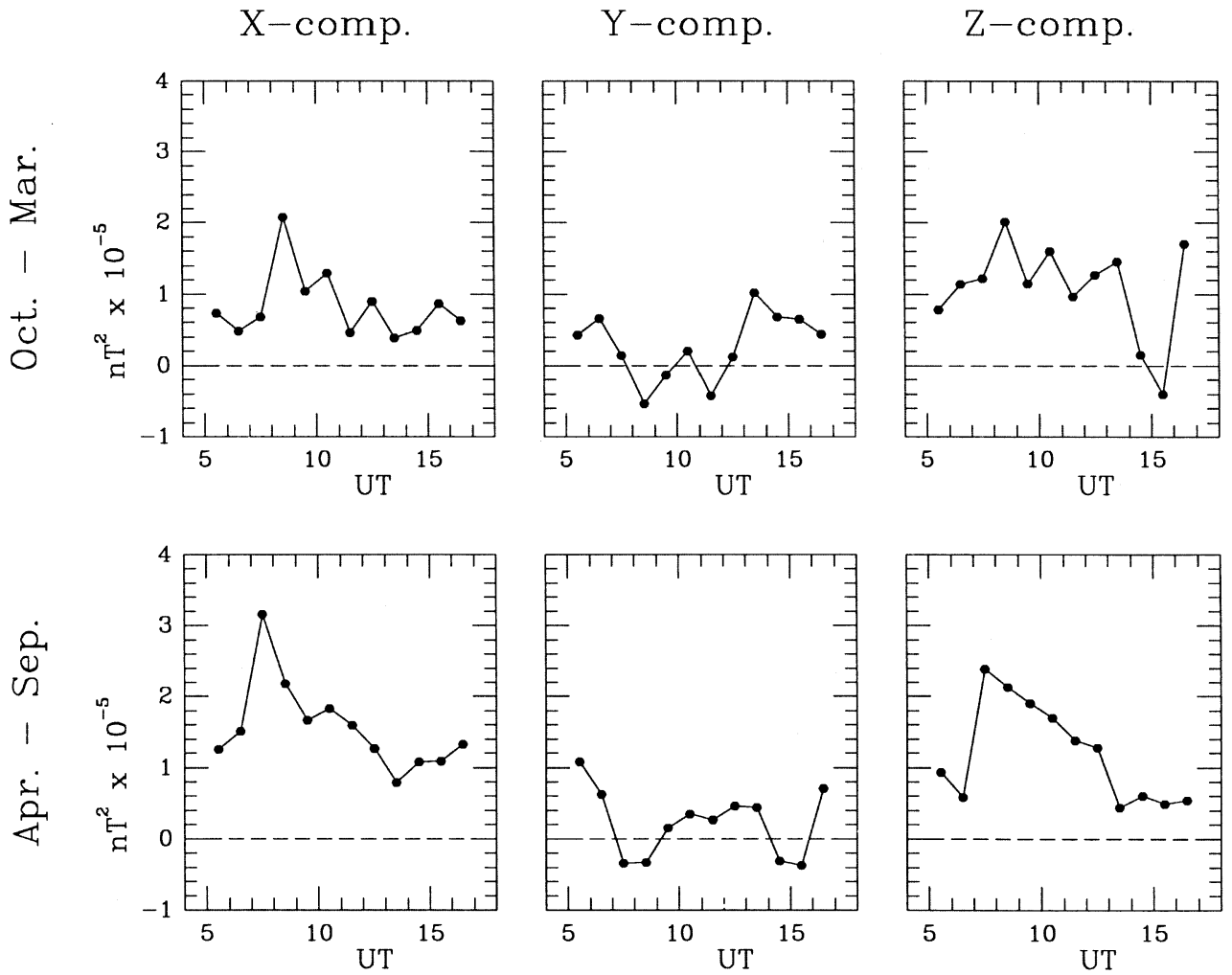

Fig. 4. Diurnal variation of the difference between the working days (Tuesday through Friday) average power and the Sunday average power in the $P c 2$ band for winter (upper panels) and summer (lower panels) months.

\section{Summary and discussion}

A long term statistical analysis conducted approximately over a solar cycle interval (1985-1994) allowed us to investigate, with high statistical confidence, some effects of local man-made disturbances on the experimental measurements in the approximate frequency range of micropulsations $(4-500 \mathrm{mHz})$. In order to detect a possible contamination from artificial signals, among other shorter and longer term effects which might find correspondence also in natural variations, we analyzed the weekly modulation of the micropulsation power which should be mostly related to man-made noise. The principal conclusions of the present investigation can be summarized as follows:

a) Previous analysis of man-made disturbances in the same frequency range were conducted by Vero (1986) and by Tsirs and Loginov (1985) who found respectively a Saturday maximum and a Thursday minimum in $P c 1$ and $P i 2$ activity. Our results conflict with those observations in that, as is reasonable to expect, we found clear evidence for minimum power values during weekends, more explicitly on Sundays. Some evidence for reduced Monday morning power and for a daily-light-saving time effect is also supported by the present investigation.

b) These effects can be ascribed to some systematic man-made noise characteristic of 
working days. At our station, this working day noise can provide a significant contribution to the total local signal power in the $50-500 \mathrm{mHz}$ band. In this frequency range, the average excess of power from Tuesday through Friday is of the order of $10-15 \%$ for the $X$ and $Z$ components. Further investigation is in progress to identify the main sources of the artificial noise at our station and to understand the reasons for the different effect observed on the two horizontal components.

c) Recent investigations (Breus et al., 1995) reported a tentative correspondence between some biological variations and the geomagnetic activity on time scales between a few hours and several years. The results of our analysis may be important in this context in that they suggest that in industrial and densely populated areas the artificial signal in the micropulsation band might be much more important than that in the present case and then comparable or even greater than the natural signal. So any possible association of biological rythms with micropulsation powers (if any) should be better related with the effect of artificial signals (Ptitsyna et al., 1996). In this sense it is interesting that Villoresi et al. (1994), who conducted measurements of manmade magnetic fields in the centre of St. Petersburg during 1991 and 1994 in the frequency range $5 \mathrm{mHz}-10 \mathrm{~Hz}$ (mainly produced by direct-current trams, trains and power devices) found that the amplitude of such disturbances is very often much greater than that of natural signals during strong geomagnetic storms and shows a clear decrease during weekends.

d) In addition to this, the observed weekly modulation may well determine the appearance of spurious peaks in the spectral analysis of the amplitude variations of the micropulsation power. This is an important point in that several investigations (Saito, 1969; Halberg et al., 1991) showed that the micropulsation power, like many other solar wind and magnetospheric parameters, shows a clear modulation related with the period of the solar rotation and some evidence for higher harmonics of the 27-day modulation has been recently proposed for the geomagnetic activity indices by Clua de Gonzalez et al. (1993).

\section{Acknowledgements}

This research activity was supported by MURST - LEMI project.

\section{REFERENCES}

Breus, T.K., G. Cornelissen, F. Halberg and A.E. LEVITIN (1995): Temporal associations of life with solar and geophysical activity, Ann. Geophysicae, 13, 1211-1222.

Cantarano, S., P. Cerulli-Irelli, A. Egidi, R. Orfei, M. Vellante and U. Villante (1983): A facility for measuring geomagnetic micropulsations at L'Aquila, Italy, Nuovo Cimento, 6C, 40-48.

Clua de Gonzalez, A.L., W.D. Gonzalez, S.L.G. DuTRA and B.T. TSURUTANI (1993): Periodic variation in the geomagnetic activity: a study based on the $A_{p}$ index, J. Geophys. Res., 98, 9215-9231.

Fraser-Smith, A.C., A. Bernardi, P.R. MCGILl, E. LADD, R.A. Helliwell and O.G. Villard (1990): Low frequency magnetic field measurements near the epicenter of the $M_{s}=7.1$ Loma Prieta earthquake, Geophys. Res. Lett., 17, 1465-1468.

Fraser-Smith, A.C., P.R. MCGill, R.A. Helliwell and O.G. VILLARD (1994): Ultra-low frequency magnetic field measurements in Southern California during the Northridge earthquake of 17 January 1994, Geophys. Res. Lett., 21, 2195-2198.

Halberg, F., T.K. Breus, G. Cornelissen, C. Bingham, D.C. Hillman, J. Rigatuso, P. Delmore and E. BAKKEN (1991): International Womb-to-Tomb Chronome Initiative Group. Chronobiology in space, University of Minnesota/Medtronic Chronobiology Seminar Series 1.

JACOBS, J.A. (1970): Geomagnetic micropulsations, in Physics and Chemistry in Space, edited by J.G. ROEDER (Springer-Verlag), vol. 1.

Meloni, A., L.V. MEDFORD and L.J. LANZEROTTI (1985): Geomagnetic anomaly detected at hydromagnetic wave frequencies, J. Geophys. Res., 90, 3569-3574.

Molchanov, O.A., Yu. A. KopytenKo, P.M. Voronov, E.A. Kopytenko, T.G. Matiashvili, A.C. FraserSMITH and A. BERNARDI (1992): Results of magnetic field measurements near the epicenters of the Spitak $\left(M_{s}=6.9\right)$ and Loma Prieta $\left(M_{s}=7.1\right)$ earthquakes: comparative analysis, Geophys. Res. Lett., 19, 14951498 .

PILIPENKO, V.A. and E.N. FEDOROV (1993): Magnetotelluric sounding of the crust and hydromagnetic monitoring of the magnetosphere with the use of ULF waves, Ann. Geofis., 36, 19-33.

Ptitsyna, N.G., G. Villoresi, Y.A. Kopytenko, V.A. Kudrin, M.I. Tyasto, E.A. KopytenKo, N. IUCCI, P.M. VORONOV and D.B. ZAITSEV (1996): Coronary heart diseases: an assessment of risk associated with work exposure to ultra-low frequency magnetic fields, Bioelectromagnetics, 17, 436-444.

SAITO, T. (1969): Geomagnetic pulsations, Space Sci. Rev., 
SAMSON, J.C. (1991): Geomagnetic pulsations and plasma waves in the Earth's magnetosphere, in Geomagnetism, edited by J.A. JACOBS (Academic Press), vol. 4.

TsiRs, G.P. and G.A. LoGINOV (1985): Peculiarities of week variations of $P c 1$ and $P i 2$ geomagnetic pulsations, Geomagn. Aeron., 25, 165-166.

Vellante, M., U. Villante, M. De Lauretis and G. BARCHI (1996): Solar cycle variation of the dominant frequencies of $P c 3$ geomagnetic pulsations at $L=1.6$, Geophys. Res. Lett., 23, 1505-1508.

VERO, J. (1986): Experimental aspects of low-latitude pulsations - a review, J. Geophys., 60, 106-119.

Villante, U. and M. VellaNTE (1997): Experimental aspects of low latitude ground pulsations, in Solar System
Plasma Physics, Conference Proceedings, edited by F. MARIANI and N.F. Ness (SIF, Bologna), vol. 56, 189-206.

Villante, U., M. Vellante, M. De lauretis, P. Cerulli-Irelli, L.J. LANZerotti, L.V. MedFord and C.G. MACLENNAN (1998): Surface and underground measurements of geomagnetic variations in the micropulsations band, Geophys. Prospect., 46, 121-140.

Villoresi, G., Y.A. Kopytenko, N.G. Ptitsyna, M.I. TYASTO, E.A. KopYTENKO, N. IUCCI and P.M. VORONOV (1994): The influence of geomagnetic storms and man-made magnetic field disturbances on the incidence of myocardial infarction in St. Petersburg (Russia), Physica Medica, 4, 107-117. 